\title{
EFFECT OF IMAGE PROCESSING OF A LEAF PHOTOGRAPH ON THE CALCULATED FRACTAL DIMENSION OF LEAF VEINS
}

\author{
Yun Kong ${ }^{1}$, Shaohui Wang ${ }^{1}$, Chengwei Ma ${ }^{2}$, Baoming $\mathrm{Li}^{2}$, Yuncong Yao ${ }^{1, *}$ \\ ${ }^{1}$ Department of Plant Science \& Technology, Beijing Agricultural College, Beijing, \\ 102206, China \\ ${ }^{2}$ Key Laboratory of Bioenvironmental Engineering of Ministry of Agriculture, College of \\ Hydraulic and Civil Engineering, China Agricultural University, Beijing, 100083, China \\ * Corresponding Author, Address: Department of Plant Science \& Technology, Beijing \\ Agricultural College, Beijing, 102206, China, Tel: +86-10-80799000, Fax: +86-10- \\ 80799000, Email:ky0257@126.com
}

Abstract: Digital photography is a promised method for estimating the fractal characteristics of leaf veins. In this study, the effects of different threshold levels and image processing methods using Adobe Photoshop software on the fractal dimension values were examined from a digital photo of nectarine leaf. The results showed that the nectarine leaf vein has typical fractal characteristics and its fractal dimension increased linearly with the increasing levels of threshold. A larger value of fractal dimension was calculated from the image processed by dark strokes, and a smaller value by accented edges or desaturate. A positive relationship was found between the calculated values of fractal dimension and the black coverage in the image. Therefore, it should be cautious to choose threshold levels and image processing methods when processing a digital image for calculating the fractal characteristics of leaf veins.

Keywords: image processing, threshold, leaf vein, fractal dimension

\section{INTRODUCTION}

Veins of some leaves have typical fractal characteristics. Their boxcounting dimension calculated by some fractal software can be used for

Kong, Y., Wang, S., Ma, C., Li, B. and Yao, Y., 2008, in IFIP International Federation for Information Processing, Volume 259; Computer and Computing Technologies in Agriculture, Vol. 2; Daoliang Li; (Boston: Springer), pp. 755-760. 
plant classification (Liu et al., 2005; Sasaki et al., 1994). But it took much time to prepare the leaf samples used for scanning photos of leaf veins, because of the difficulty of retaining only leaf veins and removing other leaf parts. With the development of digital camera and image processing, a clear digital photo of leaf can be non-destructively obtained by some image processing software such as Adobe Photoshop after capturing it in the filed. Then the processed photo can be directly used for fractal analysis of leaf veins. However, the information is meager for the effect of image processing method on the calculated result of fractal dimension of leaf vein.

The objective of the paper is to study how fractal dimension values change with the different threshold levels, and the different image processing methods.

\section{MATERIAL AND METHOD}

A color photograph of lower side of leaf in a nectarine tree (Fig. 1), which was grown inside a chinese type lean-to greenhouse in the horticultural experiment station at Beijing Agricultural College, was taken in a still and overcast sky condition using a digital camera (DSC-F717, Sony Corporation, Japan) on May 12th, 2006. The effective pixel count was 5.0 million pixels. An automatic setting for the aperture width and shutter speed was used. The characteristics of the image were $2048 \times 1536$ Pixel in resolution, 1.20M in size and JPG file in type.

The photograph was downloaded directly from the digital camera to a personal computer and then was analyzed by the following steps of imageprocessing using Adobe Photoshop software for Windows (Adobe Systems Inc., San José, CA, USA). Step 1, with the newly opened image visible in a Adobe Photoshop window, select Image $>$ Adjust $>$ Invert, and then choose Image $>$ Adjust $>$ Threshold from the menu bar. The threshold was set at seven values ranging from $85-115$ every five levels. Then, click on OK to produce 7 different quality binary images and saved them as BMP files (Fig. 2). Step 2, after opening one of the above saved files with the threshold

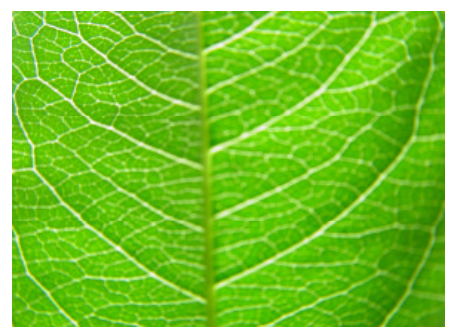

Figure 1. A color digital photograph of lower side of nectarine leaf 
level of 100, select Filter $>$ Brush Strokes $>$ Accented Edges, or $>$ Dark Strokes and the save them as two different processed images (Fig. 3). Step 3, after opening and inverting the photo, follow the Step 1, choose Image $>$ Adjust $>$ Desaturate or Filter $>$ Brush Strokes $>$ Dark Strokes, and then select Image $>$ Adjust $>$ Threshold, and set the level at 100. Click on OK to produce 2 different-quality binary images (Fig. 4).

All of the above black-white photos were then be used for calculating fractal dimension of leaf veins by a Fractal Analysis Software (Sasaki etal., 1994), developed by Hiroyuki Sasaki (Grassland Subteam for Integrated Soil Fertility Management, National Institute of Livestock and Grassland Science, Nasushiobara, Tochigi, Japan). The detailed procedure is described in its user manual.

\section{RESULT}

As found in Fig. 2, with the increment in the levels of threshold, the minor leaf veins became clear and at the same time the edges of some leaf veins became obscure.

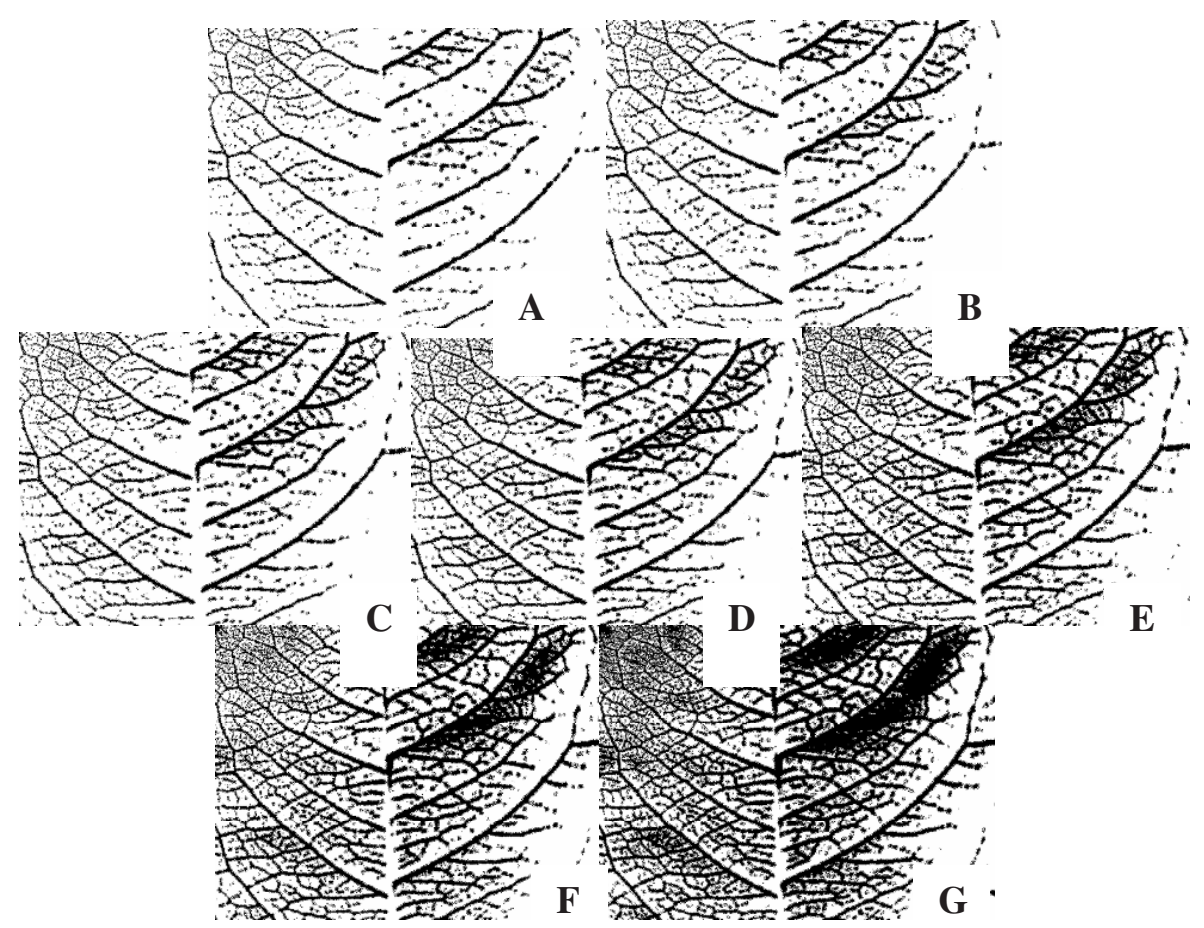

Figure 2. Effect of different threshold levels on the binary image quality

Note: The threshold levels of photo A-D were set at 85, 90, 95, 100, 105, 110, 115 respectively 
The nectarine leaf veins have typical fractal characteristics due to a large correlation coefficient of above 0.99(Table 1). In addition, the fractal dimension presented a linear increment with the increasing levels of threshold. The regressed equation was obtained as follows: $\mathrm{y}=0.0086 \mathrm{x}+$ $0.8617\left(\mathrm{R}^{2}=1\right)$.

Table 1. Effect of different threshold levels on the fractal dimension of leaf veins

\begin{tabular}{lccc}
\hline Threshold level & Cover $(\%)$ & $\mathrm{R}(\mathrm{n})$ & Fractal dimension \\
\hline 85 & 11.7 & $-0.9983(10)$ & 1.5966 \\
90 & 14.8 & $-0.9985(10)$ & 1.6394 \\
95 & 18.6 & $-0.9988(10)$ & 1.6821 \\
100 & 23.3 & $-0.9990(10)$ & 1.727 \\
105 & 29 & $-0.9993(10)$ & 1.7695 \\
110 & 36 & $-0.9995(10)$ & 1.8124 \\
115 & 45 & $-0.9997(10)$ & 1.8555 \\
\hline
\end{tabular}

Note: cover is the percent of black coverage in the processed image, $\mathrm{R}$, the correlation coefficient, and $\mathrm{n}$, the number of data to calculate.

After the binary images were processed by accented edges and dark strokes, it was difficult to find the significant changes by eye (Fig. 3). But from Table 2, a less value of fractal dimension could be found after being processed by accented edges, and a larger one by dark strokes.

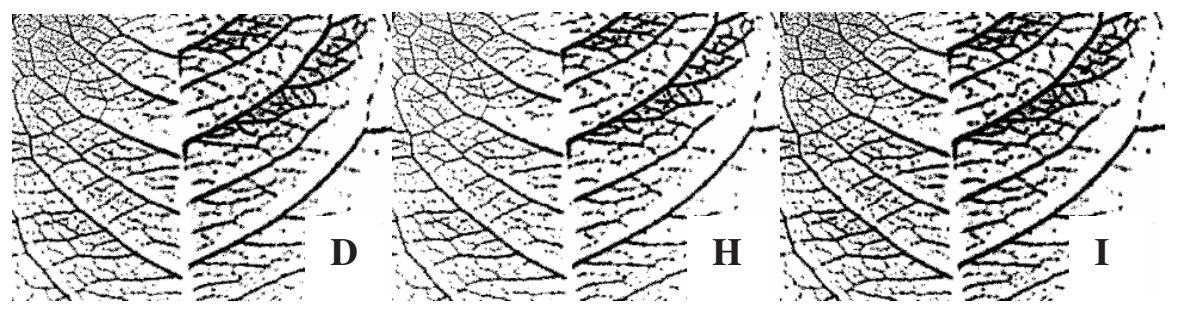

Figure 3. Effect of different filter processing methods including accented edges and dark strokes on the binary image quality

Note: The photos $\mathrm{H}$ and $\mathrm{D}$ were processed by accented edges and dark strokes respectively

Table 2. Effect of different filter processing methods including accented edges and dark strokes on the fractal dimension of leaf veins

\begin{tabular}{lccc}
\hline Image number & Cover $(\%)$ & $\mathrm{R}(\mathrm{n})$ & Fractal dimension \\
\hline $\mathrm{D}$ & 23.3 & $-0.9990(10)$ & 1.727 \\
$\mathrm{H}$ & 19.2 & $-0.9987(10)$ & 1.6861 \\
$\mathrm{I}$ & 27.3 & $-0.9993(10)$ & 1.756 \\
\hline
\end{tabular}


Before being converted into a binary image, the photo was processed by desaturate and dark strokes produced two different-quality white-black images respectively although both threshold levels were set at 100 (Fig. 4). The former had a remarkably less black coverage, while the latter had a significantly more one. Associated with this, as seen in Table 3, the photo was processed by desaturate before being converted into a binary image had a less value of fractal dimension, and it had been done by dark strokes with a larger value of that.
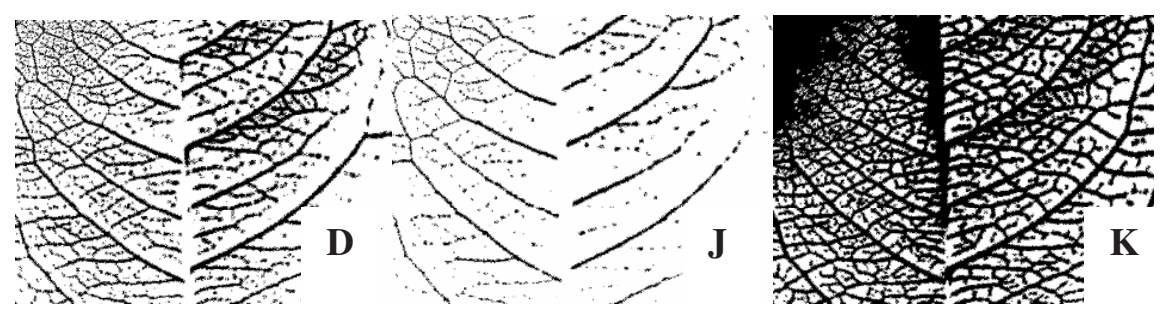

Figure 4. Effect of processing methods of desaturate and dark strokes before converting into binary image on the ultimate image quality

Note: The photos $\mathrm{J}$ and $\mathrm{K}$ were processed by desaturate and dark strokes respectively

Table 3. Effect of processing methods of desaturate and dark strokes before converting into binary image on the fractal dimension of leaf vein

\begin{tabular}{lccc}
\hline Image number & Cover $(\%)$ & $\mathrm{R}(\mathrm{n})$ & Fractal dimension \\
\hline $\mathrm{D}$ & 23.3 & $-0.9990(10)$ & 1.727 \\
$\mathrm{~J}$ & 7.2 & $-0.9977(10)$ & 1.5007 \\
$\mathrm{~K}$ & 57.9 & $-0.9998(10)$ & 1.8987 \\
\hline
\end{tabular}

\section{DISCUSSION}

From all the tables above, it could be found that larger values of fractal dimension were associated with the increment of black coverage in the image. There was a logarithmic relationship between the fractal dimension value and the percentage of the black coverage (Fig. 5). 


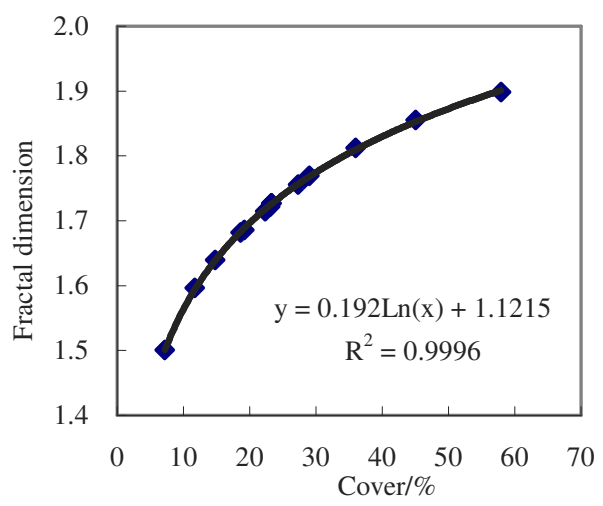

Figure 5. The relationship between the calculated fractal dimension and black cover in the processed images

\section{CONCLUSION}

Different threshold levels and different image processing methods produced different black coverage in the image and then resulted in different values of fractal dimension of leaf veins. Therefore, when calculating the fractal dimension, the images should be processed by the same methods and the same threshold levels in order to compare the fractal dimension of different images.

\section{ACKNOWLEDGEMENTS}

The authors are indebted to the cooperation program between Beijing Education Committee and China Agricultural University (XK100190553) for the financial support.

\section{REFERENCES}

Adobe Systems Inc. Adobe Photoshop version 6.0. San Jose, CA. 2001.

Liu T., Zou X., Zhao T., et al. Fractal characteristics of leaves of Osynanthus fragrans lour. Journal of Wuhan Botanical Research, 2005, 23(2):199-202.

Sasaki H., S. Shibata, T. Hatanaka. An evaluation method of ecotypes of Japanese lawn grass (Zoysia japonica STEUD.) for three different ecological functions. Bull. Natl. Grassl. Res. Inst. 1994, 49:17-24. 